\title{
A Regularized Clustering Algorithm Based on Calculus of Variations
}

\author{
Benson S. Y. Lam ${ }^{1}$, Alan Wee-Chung Liew ${ }^{2}$, David K. Smith ${ }^{3}$ and Hong Yan ${ }^{1,4}$ \\ ${ }^{1}$ Department of Electronic Engineering, City University of Hong Kong, Kowloon, Hong Kong \\ ${ }^{2}$ School of Information and Communication Technology \\ Griffith University, Queensland, Australia \\ ${ }^{3}$ Department of Biochemistry, University of Hong Kong, Pok Fu Lam, Hong Kong \\ ${ }^{4}$ School of Electrical and Information Engineering, University of Sydney, NSW 2006, Australia \\ E-mail: benson.lam@,student.cityu.edu.hk, a.liew@griffith.edu.au,h.yan@cityu.edu.hk
}

\begin{abstract}
Microarray data clustering has drawn great attention in recent years. However, a major problem in data clustering is convergence to a local optimal solution. In this paper, we introduce a regularized version of the $l_{2 m}-F C M$ algorithm to resolve this problem. The strategy is to constrain the descent direction in the optimization procedure. For this we employ a novel method, calculus of variations, to correct the direction. Experimental results show that the proposed method has a better performance than seven other clustering algorithms for three synthetic and six real world data sets. Also, the proposed method produces reliable results for synthetic data sets with a large number of groups, which is a challenging problem for many clustering algorithms. Our method has been applied to microarray data classification with good results.
\end{abstract}




\section{Introduction}

Microarray data analysis is useful for understanding biological processes based on patterns of gene expression. Genes, which have similar expression patterns, are more likely to be coregulated and may have common functions. Early examples were the study of yeast sporulation, which consists of meiosis and spore morphogenesis, by Chu et al. [1] and that of Cho et al. who used a similar technique to study the mitotic cell cycle [2]. More recently Agrawal used more sophisticated techniques to analyze a cancer data set based on measuring the similarity among genes [3]. In general, a microarray data set can be viewed as an $n \times d$ matrix, where $n$ is the number of genes and $d$ is the number of features (conditions or experiments). Usually, $n$ is a very large number, in the order of thousands and $d$ is relatively small. If the microarray data is analyzed manually, the procedure will be very labor intensive. This leads to the need for automated clustering by computer. Many clustering algorithms have been proposed including the $k$-means, $k$-medoids, fuzzy c-means, and hierarchical clustering algorithms [4-7]. A comprehensive review of clustering methods can be found in [8-11]. Although these algorithms can produce good results, they have a common limitation that they can converge to a local optimum and so produce less reliable results. There are several reasons for this. The data set may contain noise or outliers [12] and these data points can make a very large contribution to the objective function and thus, may be classified as a group. Also, the objective function itself may contain local optima. Genetic algorithms and simulated annealing methods are robust in finding global optimal solutions [13-15], but they can be very time consuming.

In this paper, we propose a new method to avoid local optimal solutions by correcting the descent direction. This is based on calculus of variations [16]. Calculus of variations has been widely used in mechanics to solve optimisation problems [17]. In order to avoid local optima caused by noise and outliers in the data sets, we apply this technique to our recently developed clustering algorithm, the modified $l_{2}$ norm based FCM algorithm $\left(l_{2 \mathrm{~m}}-\mathrm{FCM}\right.$ algorithm) [18]. The $l_{2 \mathrm{~m}}$-FCM algorithm is robust against noise and outliers. It locates the medians of the data set. Experiment results show that the performance of the $l_{2 \mathrm{~m}}-\mathrm{FCM}$ algorithm is greatly enhanced by the addition of our method to avoid local optima. The proposed method can obtain reliable results for data sets with a large number of groups, which is a challenging problem for many clustering algorithms. We have compared the proposed method with seven other clustering algorithms using both synthetic and real world data sets and found that the proposed method generates better results. Our preliminary results have been presented in [19].

The organization of the paper is as follows. In Section 2, we review the $l_{2 \mathrm{~m}}-\mathrm{FCM}$ algorithm. Then, we correct the descent direction by proposing a new weighting function based on the calculus of variations in Section 3. The robustness of the proposed method is shown by experiments in Section 4. Finally, our conclusion is given in Section 5.

\section{The $I_{2 m}-F C M$ Algorithm}

Assume the input data samples are $\mathbf{X}=\left\{\mathbf{x}_{1}, \mathbf{x}_{2}, \ldots \mathbf{x}_{\mathrm{n}}\right\}$. The data samples are divided into $c$ classes and each class is represented by a cluster center $\mathbf{v}_{\mathrm{k}} \in \mathrm{V}=\left\{\mathbf{v}_{1}, \ldots, \mathbf{v}_{\mathrm{c}}\right\}$ where $1 \leq k \leq c$. Let $\mathrm{U}=\left\{\mu_{\mathrm{ik}}, 1 \leq i \leq n, 1 \leq k \leq c\right\}$, where $\mu_{\mathrm{ik}}$ is the membership value of $\mathbf{x}_{\mathrm{i}}$ belonging to cluster $k$ and satisfying the following two conditions: 


$$
\begin{gathered}
1 \leq i \leq n \\
0<\mu_{\mathrm{ik}}<1 \quad \quad \quad \sum_{k=1}^{c} \mu_{i k}=1 \quad 0 \leq i \leq n .
\end{gathered}
$$

In the $l_{2 \mathrm{~m}}-\mathrm{FCM}$ algorithm, we minimize the following objective function:

$$
\mathrm{J}(\mathrm{U}, \mathrm{V} ; \mathrm{X})=\sum_{i=1}^{n} \sum_{k=1}^{c} \mu_{i k}^{m}\left\|\mathbf{x}_{i}-\mathbf{v}_{k}\right\|_{\varepsilon},
$$

where $\|\mathbf{u}\|_{\varepsilon}=\sqrt{\varepsilon^{2}+\|\mathbf{u}\|_{2}^{2}}$. The use of parameter $\varepsilon$ in the norm is to avoid the derivative of $\mathbf{J}$ with respect to $\mathbf{v}_{\mathrm{k}}$ being zero. The value of $\varepsilon$ should be very small to avoid numerical error. In Appendix A, we prove the case that if $c=1$, the difference between the solutions by $\mathrm{J}$ at $\varepsilon \neq 0$ and $\mathrm{J}$ at $\varepsilon=0$ is within $2 \varepsilon$. The update step equations are

$$
\begin{gathered}
\mu_{i k}=\frac{\left\|\mathbf{x}_{i}-\mathbf{v}_{k}^{(p-1)}\right\|_{\varepsilon}^{-1 /(m-1)}}{\sum_{k=1}^{c}\left\|\mathbf{x}_{i}-\mathbf{v}_{k}^{(p-1)}\right\|_{\varepsilon}^{-1 /(m-1)}} \text { and } \\
\mathbf{v}_{k}^{(p)}=\frac{\sum_{i=1}^{n} \mu_{i k}^{m}\left(\frac{\mathbf{x}_{i}}{\left\|\mathbf{v}_{k}^{(p-1)}-\mathbf{x}_{i}\right\|_{\varepsilon}}\right)}{\sum_{i=1}^{n} \mu_{i k}^{m}\left(\frac{1}{\left\|\mathbf{v}_{k}^{(p-1)}-\mathbf{x}_{i}\right\|_{\varepsilon}}\right)} .
\end{gathered}
$$

Equation (4) is a nonlinear equation for the unknown $\mathbf{v}_{\mathrm{k}}$ and it is possible for it to converge to a local optimum. We consider a data set with 15 groups and its clustering results using the $l_{2 \mathrm{~m}^{-}}$ FCM algorithm are shown in Figure 1. The cluster centers are displayed as large gray dots. No cluster center assigned to the two groups at the bottom and one cluster center is moved to the noise region placed at the right hand side of the figure.

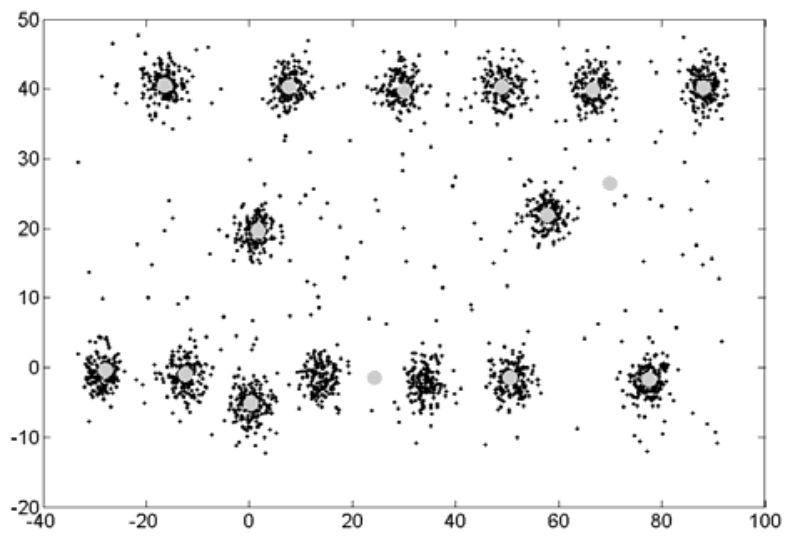

Figure 1. Clustering result using $l_{2 \mathrm{~m}}$-FCM algorithm for a data set with 15 groups.

We investigate the equations by substituting Equation (3) into Equation (4). Taking $m=1.5$, we have 


$$
\begin{gathered}
\mathbf{v}_{k}^{(p)}=\frac{\sum_{i=1}^{n} \mathbf{x}_{i}\left\|\mathbf{v}_{k}^{(p-1)}-\mathbf{x}_{i}\right\|_{\varepsilon}^{-4} / z_{i}}{\sum_{i=1}^{n}\left\|\mathbf{v}_{k}^{(p-1)}-\mathbf{x}_{i}\right\|_{\varepsilon}^{-4} / z_{i}}, \\
\text { where } z_{i}=\left(\sum_{k=1}^{c}\left\|\mathbf{v}_{k}^{(p-1)}-\mathbf{x}_{i}\right\|_{\varepsilon}^{-2}\right)^{1.5} .
\end{gathered}
$$

For simplicity, we denote a point close to $\mathbf{v}_{\mathrm{k}}$ as $\mathbf{x}_{\mathrm{c}}$ while a point far away from $\mathbf{v}_{\mathrm{k}}$ as $\mathbf{x}_{\mathrm{f}}$. In this equation, we can see that the weighting function $y=\left\|\mathbf{v}_{\mathrm{k}}-\mathbf{x}_{\mathrm{i}}\right\|^{-4}$ is extremely large if $\mathbf{x}_{\mathrm{i}}=\mathbf{x}_{\mathrm{c}}$ while $y$ is very small if $\mathbf{x}_{\mathrm{i}}=\mathbf{x}_{\mathrm{f}}$. If two cluster centers $\mathbf{v}_{\mathrm{p}}$ and $\mathbf{v}_{\mathrm{q}}$ are placed near $\mathbf{x}_{\mathrm{c}}$, their weighting functions will be very large. However, the contribution of $\mathbf{x}_{\mathrm{f}}$ is very small. If $\mathbf{x}_{\mathrm{f}}$ is a member of another group, the algorithm may converge to a local optimum.

To resolve this problem, we have to re-distribute the weighting function so that points like $\mathbf{x}_{\mathrm{c}}$ make smaller contributions while preserving $\mathbf{x}_{\mathrm{f}}$ contribution. This is explained in the next section.

\section{Method Based on Calculus of Variations}

\subsection{A New Weighting Function}

In this section, we introduce the calculus of variations [16] to correct the descent direction of the $l_{2 \mathrm{~m}}-\mathrm{FCM}$ algorithm. An introduction to the calculus of variations can be found in Appendix B.

We formulate the original $l_{2 \mathrm{~m}}-\mathrm{FCM}$ algorithm in a continuous manner and we only focus on the unknown function $\mathbf{v}(\mathrm{s})$.

$$
J(\mathbf{v}(\mathrm{s}))=\int_{\Gamma} \int_{\Omega} \mu^{m}(\mathrm{~s}, \mathrm{t})\|\mathbf{x}(\mathrm{t})-\mathbf{v}(\mathrm{s})\|_{\varepsilon} H(\mathrm{~s}) \mathrm{dsdt}
$$

where $\Omega=[0,1], \Gamma$ is the domain of the data set $\mathbf{X}$ and $\mathrm{H}(\mathrm{s})$ is a delta function. $\mathrm{H}(\mathrm{s}) \rightarrow \infty$ if $\mathrm{s} \in\left\{\mathrm{s}_{\mathrm{k}}\right.$ : $\left.0 \leq \mathrm{s}_{\mathrm{k}} \leq 1, k=1,2, \ldots c\right\}$, otherwise, $\mathrm{H}(\mathrm{s})=0$. We apply the calculus of variations to this objective function with a function $\varphi(s, t)$, which means the perturbation is dependent on data $\mathbf{X}$. Taking the derivative with respect to $\gamma$ at $\gamma=0$, we have

$$
\begin{aligned}
\left.\frac{\mathrm{d}}{\mathrm{d} \gamma}\right|_{\gamma=0} J(\mathbf{v}(\mathrm{s})+\gamma \varphi(\mathrm{s}, \mathrm{t})) & =\left.\frac{\mathrm{d}}{\mathrm{d} \gamma}\right|_{\gamma=0} \int_{\Gamma} \int_{\Omega} \mu^{m}(\mathrm{~s}, \mathrm{t})\|\mathbf{x}(\mathrm{t})-\mathbf{v}(\mathrm{s})-\gamma \varphi(\mathrm{s}, \mathrm{t})\|_{\varepsilon} \mathrm{H}(\mathrm{s}) \mathrm{dsdt} \\
& =\iint_{\Gamma} \mu_{\Omega}^{m}(\mathrm{~s}, \mathrm{t}) \frac{\mathbf{v}(\mathrm{s})-\mathbf{x}(\mathrm{t})}{\|\mathbf{v}(\mathrm{s})-\mathbf{x}(\mathrm{t})\|_{\varepsilon}} \varphi(\mathrm{s}, \mathrm{t}) \mathrm{H}(\mathrm{s}) \mathrm{dsdt}
\end{aligned}
$$

We correct the search direction by the following setting. $\varphi(s, t)$ is taken to be the composition of two functions. i.e. $\varphi(\mathrm{s}, \mathrm{t})=\phi(\mathrm{s}, \mathrm{t}) \psi(\mathrm{s}) \cdot \psi(\mathrm{s})$ is an arbitrary function while $\phi(\mathrm{s}, \mathrm{t})$ is a function satisfying the following three conditions. (1) $\phi(\mathrm{s}, \mathrm{t}) \rightarrow 0$ if $\|\mathbf{v}(\mathrm{s})-\mathbf{x}(\mathrm{t})\|_{\varepsilon} \rightarrow 0$; (2) $\phi(\mathrm{s}, \mathrm{t}) \rightarrow 1$ if $\|\mathbf{v}(\mathrm{s})-\mathbf{x}(\mathrm{t})\|_{\varepsilon} \rightarrow \infty$ and (3) For each $\mathrm{s}_{\mathrm{k}}$, the function $\mathrm{f}_{\mathrm{k}}(\mathrm{t})=\phi\left(\mathrm{s}_{\mathrm{k}}, \mathrm{t}\right) /\left\|\mathbf{v}\left(\mathrm{s}_{\mathrm{k}}\right)-\mathbf{x}(\mathrm{t})\right\|_{\varepsilon}$ contains only one minimum. The first two conditions are adopted to reduce the weight for $\mathbf{x}_{\mathrm{c}}$ while preserving the weight for $\mathbf{x}_{\mathrm{f}}$. The third condition is to restrict the function so that it contains only one optimum. We found that the function $\phi(s, t)=\|\mathbf{v}(s)-\mathbf{x}(\mathrm{t})\|_{\varepsilon} /\left(\mathrm{a}+\|\mathbf{v}(\mathrm{s})-\mathbf{x}(\mathrm{t})\|_{\varepsilon}\right)$ satisfies these three conditions. Obviously, the first two conditions are satisfied. For the third condition, the function $\mathrm{f}_{\mathrm{k}}(\mathrm{t})=$ $\phi\left(\mathrm{s}_{\mathrm{k}}, \mathrm{t}\right) /\left\|\mathbf{v}\left(\mathrm{s}_{\mathrm{k}}\right)-\mathbf{x}(\mathrm{t})\right\|_{\varepsilon}=1 /\left(\mathrm{a}+\left\|\mathbf{v}\left(\mathrm{s}_{\mathrm{k}}\right)-\mathbf{x}(\mathrm{t})\right\|_{\varepsilon}\right)$ contains only one minimum for $\left\|\mathbf{v}\left(\mathrm{s}_{\mathrm{k}}\right)-\mathbf{x}(\mathrm{t})\right\|_{\varepsilon}>0$. Putting these together, Equation (7) is now rewritten as 


$$
\begin{aligned}
& \iint_{\Gamma} \mu^{m}(\mathrm{~s}, \mathrm{t}) \frac{\mathbf{v}(\mathrm{s})-\mathbf{x}(\mathrm{t})}{\|\mathbf{v}(\mathrm{s})-\mathbf{x}(\mathrm{t})\|_{\varepsilon}} \varphi(\mathrm{s}, \mathrm{t}) \mathrm{H}(\mathrm{s}) \mathrm{dsdt} \\
& =\int_{\Gamma} \int_{\Omega} \mu^{m}(\mathrm{~s}, \mathrm{t}) \frac{\mathbf{v}(\mathrm{s})-\mathbf{x}(\mathrm{t})}{\|\mathbf{v}(\mathrm{s})-\mathbf{x}(\mathrm{t})\|_{\varepsilon}} \frac{\|\mathbf{v}(\mathrm{s})-\mathbf{x}(\mathrm{t})\|_{\varepsilon}}{\mathrm{a}+\|\mathbf{v}(\mathrm{s})-\mathbf{x}(\mathrm{t})\|_{\varepsilon}} \boldsymbol{\psi}(\mathrm{s}) \mathrm{H}(\mathrm{s}) \mathrm{dsdt} \\
& =\int_{\Gamma} \int_{\Omega} \mu^{m}(\mathrm{~s}, \mathrm{t}) \frac{\mathbf{v}(\mathrm{s})-\mathbf{x}(\mathrm{t})}{\mathrm{a}+\|\mathbf{v}(\mathrm{s})-\mathbf{x}(\mathrm{t})\|_{\varepsilon}} \boldsymbol{\psi}(\mathrm{s}) \mathrm{H}(\mathrm{s}) \mathrm{dsdt}
\end{aligned}
$$

$\psi(\mathrm{s})$ is an arbitrary function and $\mathrm{H}(\mathrm{s})$ is a delta function, which is not equal to zero only at a finite number of points. Setting Equation (8) equal to zero, we have

$$
\sum_{i=1}^{n} \mu_{i k}^{m} \frac{\mathbf{v}_{k}-\mathbf{x}_{i}}{a+\left\|\mathbf{v}_{k}-\mathbf{x}_{i}\right\|_{\varepsilon}}=0,
$$

where $\mathbf{v}_{\mathrm{k}}=\mathbf{v}\left(\mathrm{s}_{\mathrm{k}}\right)$. We use an iterative scheme to find the solution of this equation by assuming that $\mathbf{v}_{\mathrm{k}}$ on the numerator is the variable at the pth iteration and that $\mathbf{v}_{\mathrm{k}}$ on the denominator is known at the (p-1)th iteration. Thus, the new update step equation becomes

$$
\mathbf{v}_{k}^{(p)}=\frac{\sum_{i=1}^{n} \mu_{i k}^{m}\left(\frac{\mathbf{x}_{i}}{a+\left\|\mathbf{v}_{k}^{(p-1)}-\mathbf{x}_{i}\right\|_{\delta}}\right)}{\sum_{i=1}^{n} \mu_{i k}^{m}\left(\frac{1}{a+\left\|\mathbf{v}_{k}^{(p-1)}-\mathbf{x}_{i}\right\|_{\delta}}\right)} .
$$

Taking $m=1.5$, the equation for updating $\mathbf{v}_{\mathrm{k}}$ is

$$
\mathbf{v}_{k}^{(p)}=\frac{\sum_{i=1}^{n} \mathbf{x}_{i} \frac{\left\|\mathbf{v}_{k}^{(p-1)}-\mathbf{x}_{i}\right\|_{\varepsilon}^{-3}}{Z_{i}\left(a+\left\|\mathbf{v}_{k}^{(p-1)}-\mathbf{x}_{i}\right\|_{\varepsilon}\right)}}{\sum_{i=1}^{n} \frac{\left\|\mathbf{v}_{k}^{(p-1)}-\mathbf{x}_{i}\right\|_{\varepsilon}^{-3}}{z_{i}\left(a+\left\|\mathbf{v}_{k}^{(p-1)}-\mathbf{x}_{i}\right\|_{\varepsilon}\right)} .}
$$

Here, we see that the major difference between the new and old equations, Equations (5) and (11), is the parameter $a$. In Equation (4), if a cluster center $\mathbf{v}_{\mathrm{k}}$ is close to a point $\mathbf{x}_{\mathrm{c}}$, the term $1 /\left\|\mathbf{v}_{\mathrm{k}}-\mathbf{x}_{\mathrm{i}}\right\|_{\varepsilon}$ will approach infinity very fast. The contribution of $\mathbf{x}_{\mathrm{c}}$ is large while other points are relatively small. For the proposed method, a new parameter $a$ is added. The term $1 /\left(a+\left\|\mathbf{v}_{\mathrm{k}}-\mathbf{x}_{\mathrm{i}}\right\|_{\varepsilon}\right)$ approaches infinity much more slowly. The contribution of $\mathbf{x}_{\mathrm{c}}$ is reduced and the algorithm is able to escape from the local minima. This is further illustrated through the data set in Figure 2(a), where a circle represents a data point while the sign “*” represents a cluster center. Figure 2(a) shows two groups, which contains a data point far away from other points. Two points are given initial guesses of cluster centers. One is near the two groups while the other one is placed exactly at the far away point. After applying the $l_{2 \mathrm{~m}}-\mathrm{FCM}$ algorithm, the cluster centers do not move and they represent the final positions. The cluster centers obtained using the proposed method is given in Figure 2(b), which is the correct result. The new method works for the following reason. In Equation (10), the cluster centers are equal to the weight $\mathrm{w}_{\mathrm{ik}}(a)$ of the data points $\mathbf{x}_{\mathrm{i}}$ and the summation over all $i$ is equal to 1 , where $\mathrm{w}_{\mathrm{ik}}(a)$ is given as follows. 


$$
w_{i k}(a)=\frac{\mu_{i k}^{m}\left(\frac{1}{a+\left\|\mathbf{v}_{k}^{(p-1)}-\mathbf{x}_{i}\right\|_{\varepsilon}}\right)}{\sum_{i=1}^{n} \mu_{i k}^{m}\left(\frac{1}{a+\left\|\mathbf{v}_{k}^{(p-1)}-\mathbf{x}_{i}\right\|_{\delta}}\right)} .
$$

When $a$ is equal to zero, the proposed method is the same as the $l_{2 \mathrm{~m}}-\mathrm{FCM}$ algorithm. As one of the cluster center is near the far away point, one of the $\mathrm{w}_{\mathrm{ik}}(0)$ values is very large and thus the algorithm will converge to a local optimal solution. When $a$ is not equal to zero, the term $1 /\left(a+\left\|\mathbf{v}_{\mathrm{k}}-\mathbf{x}_{\mathrm{i}}\right\|_{\varepsilon}\right)$ approaches infinity much more slowly and the weights for other points are larger. Figure 2(c) shows the $\mathrm{w}_{\mathrm{ik}}(0)$ and $\mathrm{w}_{\mathrm{ik}}(500)$, where circles represent the $l_{2 \mathrm{~m}}-\mathrm{FCM}$ algorithm and the "*" signs represent the proposed method. The far away point is given a very large weight in the $l_{2 \mathrm{~m}}$-FCM algorithm, but the weight is redistributed in the proposed method and this can help the clustering process to escape from local optima.

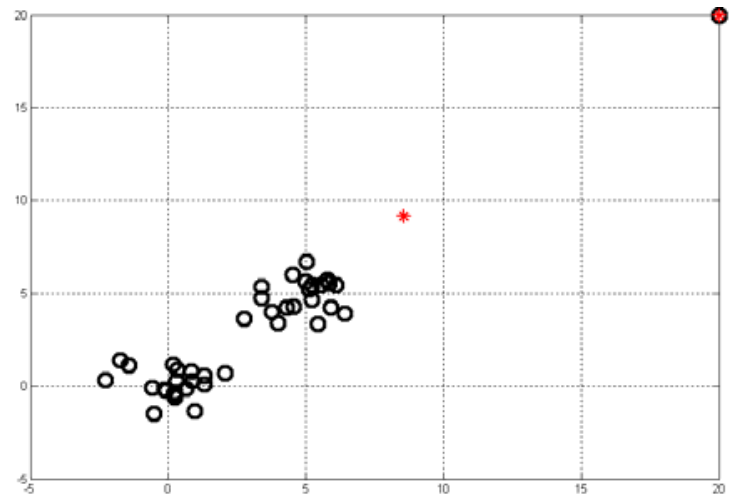

(a) A data set and the clustering result obtained using the $l_{2 \mathrm{~m}}-\mathrm{FCM}$ algorithm.

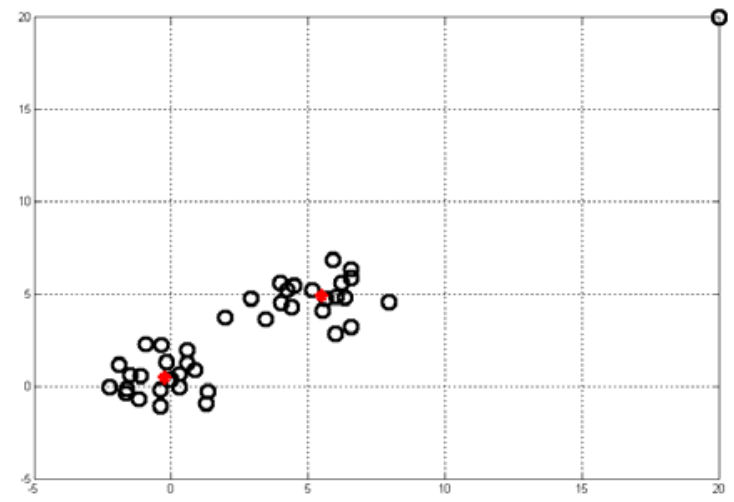

(b) Clustering result obtained using the proposed method.

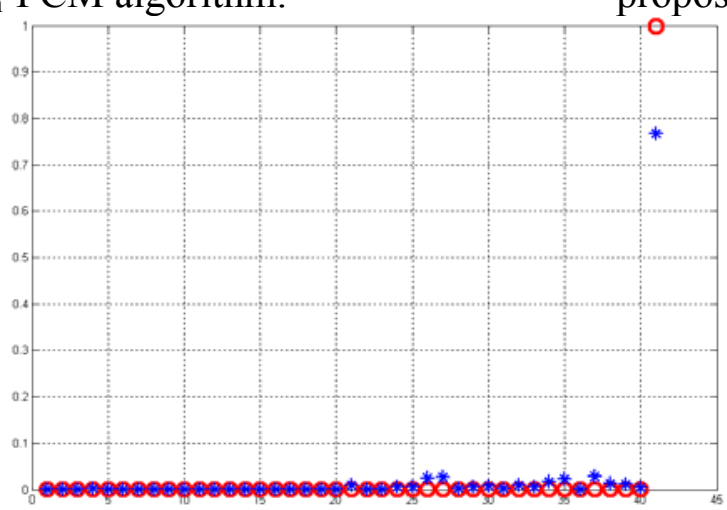

(c) The weight functions of the $l_{2 \mathrm{~m}}-\mathrm{FCM}$ algorithm and the proposed method.

Figure 2. Illustration of the proposed method.

\subsection{The Optimization Procedure}

The optimization procedure is given as follows. We gradually reduce the regularization power by decreasing the value $a$ from a high value to zero. 
Step 1: $\quad$ Initial $\mathbf{V}$ and $a$ values are given by the user.

Step 2: $\quad$ The values $\mathbf{U}$ and $\mathbf{V}$ are updated using Equations (3) and (10).

Step 3: $\quad$ Decrease $a$. If $a$ is not close to zero, go to Step 2. Otherwise, go to Step 4.

Step 4: $\quad$ The values $\mathbf{U}$ and $\mathbf{V}$ are updated using Equations (3) and (4).

In all the experiments presented in this paper, $a$ starts with the value 500 while the reduction rate is 0.9 .

\section{Experiment Results}

We show the robustness of the proposed method using three synthetic and six real world data sets with seven different clustering algorithms, including the fuzzy c-means (FCM) algorithm, the $k$-means algorithm, the alternative fuzzy c-means (Alt-FCM) algorithm [20], the Gaussian mixture model (GMM) [21], the possibilistic c-means (PCM) algorithm [22,23], the Gath-Geva algorithm (FCM-GG) [11] and the original $l_{2}$ norm based fuzzy c-means $\left(l_{2 \mathrm{~m}}\right.$-FCM) algorithm [18].

For the three synthetic data sets, their first two dimensions are given in Figures 3(a)-(c). The first data set consists of seven groups having three dimensions. Six groups contain 500 points with variance 0.5 while the one at the center has 300 points with variance one. The second data set consists of 12 groups having 3 dimensions. Eleven of them have 150 points with variance 3 while the one at the bottom has 100 points with variance 6 . The third one consists of 15 groups having 4 dimensions. Each group has 150 points with variance 2. We generate 200 noise points according to the uniform distribution. All the cluster centers for these synthetic data sets are shown in Table 1.

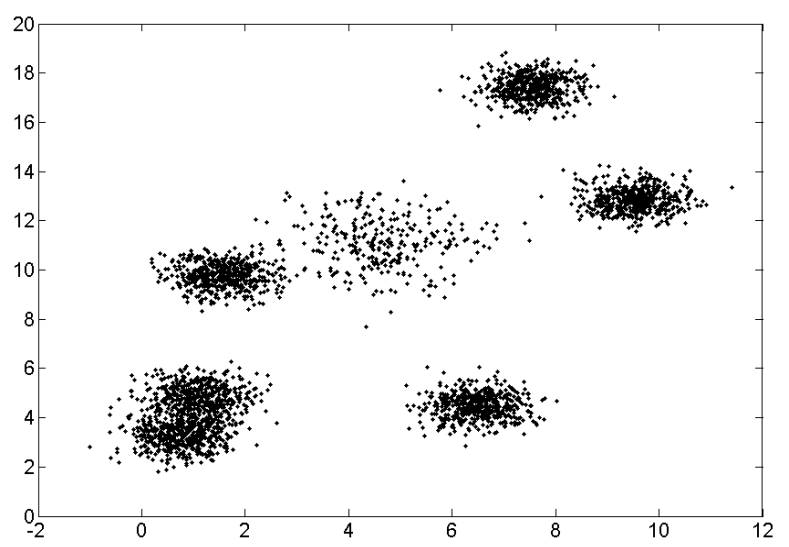

(a) Synthetic data set with seven groups.

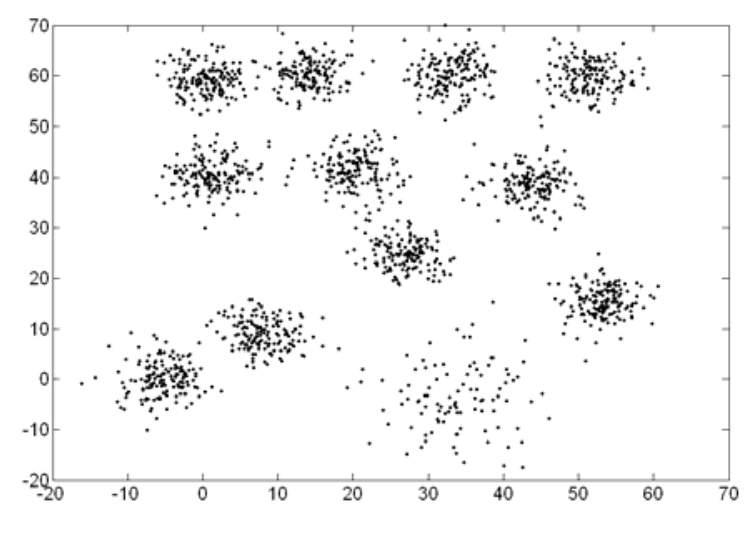

(b) Synthetic data set with 12 groups. 


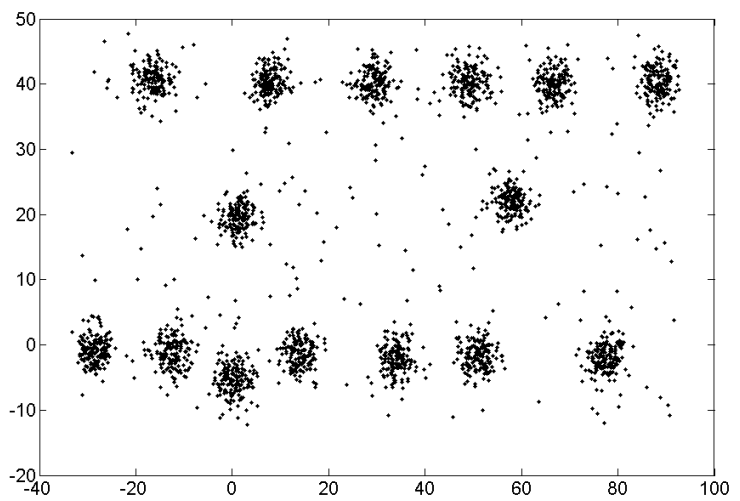

(c) Synthetic data set with 15 groups.

Figure 3. The first two dimensions of the synthetic data sets.

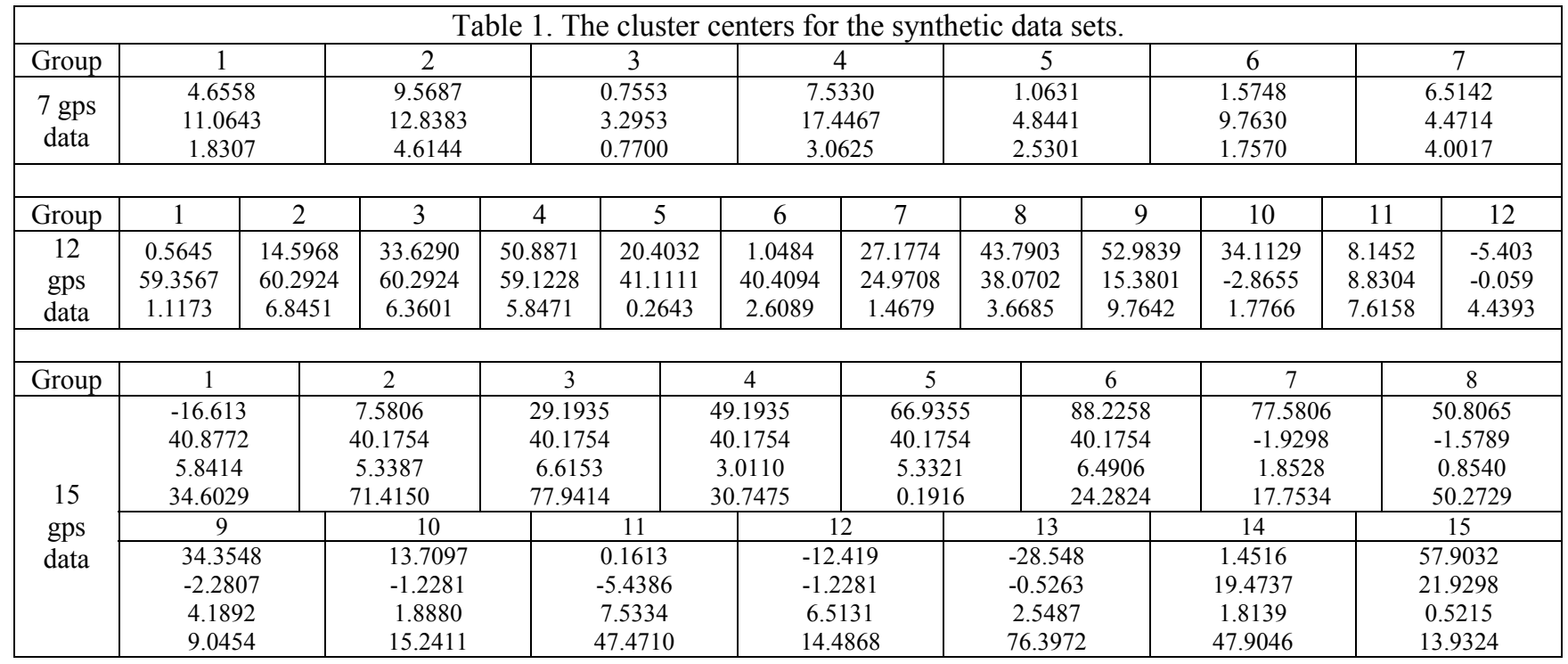

The performance measure is based on cross validation and is given below. Each data set is first randomly divided into two halves. One is for training and the other is for testing. The algorithms are run on the training set and the prototypes are obtained. Then, the testing set is used to evaluate the clustering results based on these prototypes. This process is repeated 30 times. The clustering results of these three synthetic data sets are given in Table $2 .$. In each entry of the table, there are two numbers. The number without parenthesis is the average error in percentage terms. The number in the parenthesis is the standard derivation of the error for the 30 runs. We can see that most of the algorithms converge to local optimal solutions. Only the proposed method is able to yield the same good results in these 30 runs. 


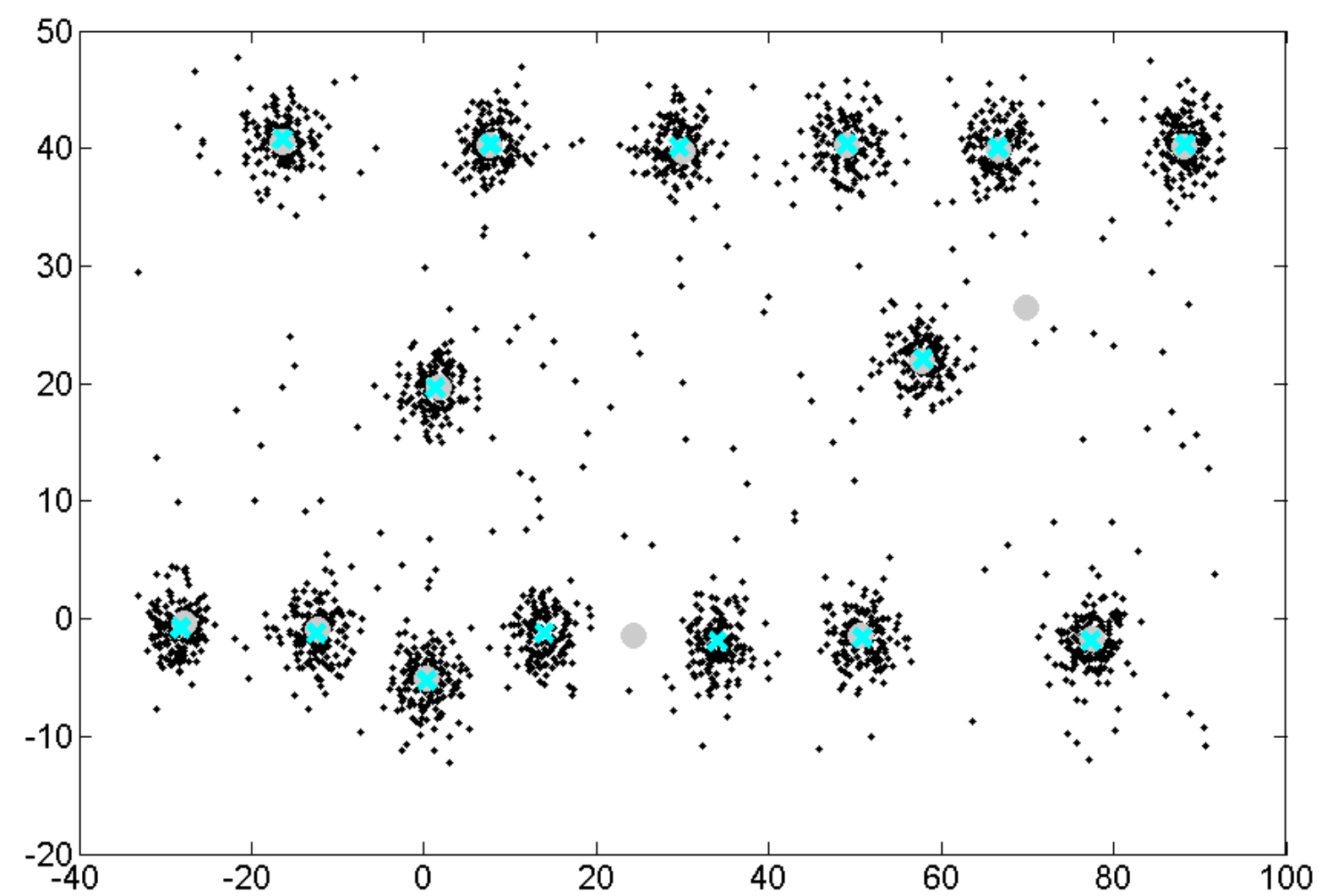

Figure 4. Clustering results using the proposed algorithm for the synthetic data set of 15 groups

( $\bullet$ is the result of $l_{2 \mathrm{~m}}-\mathrm{FCM}$ algorithm while $\times$ is the result of the proposed method.).

\begin{tabular}{|c|c|c|c|c|c|c|c|c|}
\hline \multicolumn{8}{|c|}{ Table 2. Clustering results (classification error rates) for the synthetic data sets. } \\
\hline & FCM & K-means & Alter-FCM & GMM & PCM & FCM-GG & $I_{2 \mathrm{~m}}$-FCM & Proposed \\
\hline & 14.42 & 22.50 & 13.95 & 21.83 & 10.56 & 11.62 & 12.95 & 0.24 \\
& $(9.54)$ & $(12.44)$ & $(9.83)$ & $(8.95)$ & $(9.82)$ & $(10.22)$ & $(9.85)$ & $(0.00)$ \\
\hline 7 gps data & 13.58 & 23.45 & 17.00 & 20.78 & 11.44 & 13.90 & 11.38 & 0.00 \\
& $(9.17)$ & $(9.23)$ & $(7.70)$ & $(7.35)$ & $(0.28)$ & $(9.68)$ & $(1.18)$ & $(0.00)$ \\
\hline 12 gps data & 10.42 & 28.45 & 13.08 & 23.74 & 9.67 & 11.80 & 9.65 & 0.08 \\
& $(6.25)$ & $(8.16)$ & $(7.19)$ & $(7.63)$ & $(0.41)$ & $(16.11)$ & $(5.37)$ & $(0.00)$ \\
\hline
\end{tabular}

We further test the robustness of the proposed method with four real world data sets. The iris data set (Iris) contains 150 instances, 3 classes and 4 features. The wine data set (Wine) consists of 178 instances, 3 classes and 13 features. The image data set (Image) contains 2310 instances, 10 classes and 19 features. The pen digits data set (pen digits) contains 3746 instances, 10 classes and 16 features. All these data sets are normalized to zero mean and unit variance so that each feature in the data set makes approximately the same contribution. All above data sets can be found in [24]. The clustering results for these four real world data sets are given in Table 3. In this table, we can see that in all cases the $l_{2 \mathrm{~m}}$ FCM algorithm and the proposed method yield the smallest error rate. Moreover, the proposed method has an even better performance than the original $l_{2 \mathrm{~m}}$-FCM algorithm for both image and pen digits data sets. 


\begin{tabular}{|c|c|c|c|c|c|c|c|c|}
\hline \multicolumn{8}{|c|}{ Table 3. Clustering results (classification error rates) for the real world data sets. } \\
\hline & FCM & K-means & $\begin{array}{c}\text { Alter- } \\
\text { FCM }\end{array}$ & GMM & PCM & FCM-GG & $l_{2 \mathrm{~m}}$-FCM & Proposed \\
\hline Iris & 16.00 & 16.00 & 16.00 & 19.60 & 16.00 & 26.00 & 14.70 & 14.70 \\
& $(0.00)$ & $(0.00)$ & $(0.00)$ & $(7.80)$ & $(0.00)$ & $(8.67)$ & $(0.00)$ & $(0.00)$ \\
\hline Wine & 4.55 & 8.64 & 4.55 & 26.88 & 18.18 & 27.42 & 3.41 & 3.41 \\
& $(0.00)$ & $(0.93)$ & $(0.00)$ & $(10.67)$ & $(0.00)$ & $(12.15)$ & $(0.00)$ & $(0.00)$ \\
\hline Image & 27.88 & 46.29 & 26.66 & 44.94 & 49.52 & 78.02 & 26.26 & 24.26 \\
& $(0.00)$ & $(4.19)$ & $(2.65)$ & $(5.48)$ & $(0.00)$ & $(9.21)$ & $(5.58)$ & $(4.44)$ \\
\hline Pen digits & 35.74 & 39.31 & 52.60 & 33.73 & 63.86 & 89.59 & 33.72 & 31.30 \\
& $(7.44)$ & $(6.51)$ & $(6.54)$ & $(5.64)$ & $(3.89)$ & $(0.00)$ & $(6.79)$ & $(4.45)$ \\
\hline
\end{tabular}

Next, we consider the yeast cycle and sporulation data sets published by Spellman et al. [26]. It consists of 800 genes over 17 time points taken at 10 minutes intervals. There are five phases in the yeast cell cycle data set and they are G1, S, G2, M, and M/G1. After splitting the yeast data set into two halves, there are 403 data samples for training and 397 data points for testing. In the 397 samples, the five groups contain 56, 149, 35, 60 and 97 genes respectively. The clustering results are shown in Table 4. The error rate, using our method, is the smallest at $42.32 \%$, and in each of the 30 runs, our method yields the same result. The proposed method can correctly identify 15, 124, 0, 1 and 89 genes in these five groups respectively. There are more genes correctly recognized in the $\mathrm{S}$ and $\mathrm{M} / \mathrm{G} 1$ phases. The PCM algorithm can produce the same error as the proposed method. It can correctly identify $0,137,0,0$ and 92 genes in these five groups respectively. Although the PCM algorithm can identify more genes in both S and M/G1 phases than the proposed method, it fails to detect genes in other phases.

We have also analyzed that the sporulation data published by Chu et al [26]. The data set consists of 6118 genes. We adopt the post-processing steps by Datta et al. [27]. We only take the genes, for which the root mean square value of the $\log 2$ transform of the data is greater than 1.13. After the post-processing, the data set is normalized to zero mean and unit variance and we obtain a subset of the data set, which contains 1136 genes having seven phases. The seven phases are rapid transient induction ("metabolic"), early I induction, early II induction, early-middle induction, middle induction, mid-late induction and late induction. After splitting the data set into two halves, there are 570 genes in the training set and 566 genes in the testing set. The 566 testing samples contain 87, 128, 104, 86, 91, 13 and 57 genes in the seven phases, respectively. The clustering results are given in Table 4 . The average error rate of the proposed method for 30 runs is the smallest at $58.15 \%$. At the minimum error rate $57.42 \%$ in of one of the 30 runs, the proposed method can correctly identify 55, 48, 39, 34, 26, 0 and 39 genes in these seven phases. There are more genes identified at rapid transient induction and early I induction.

\begin{tabular}{|c|c|c|c|c|c|c|c|c|}
\hline \multicolumn{8}{|c|}{ Table 4. Clustering results (classification error rates) for the two microarray data sets. } \\
\hline & FCM & K-means & $\begin{array}{c}\text { Alter- } \\
\text { FCM }\end{array}$ & GMM & PCM & FCM-GG & $l_{2 \mathrm{~m}}$-FCM & Proposed \\
\hline Yeast & 46.32 & 44.53 & 46.84 & 53.70 & 42.32 & 53.73 & 42.32 & 42.32 \\
& $(0.94)$ & $(4.32)$ & $(0.13)$ & $(9.09)$ & $(0.00)$ & $(7.61)$ & $(0.00)$ & $(0.00)$ \\
\hline Sporulation & 62.53 & 61.07 & 68.65 & 68.10 & 61.48 & 61.38 & 58.20 & 58.15 \\
& $(3.16)$ & $(3.55)$ & $(1.51)$ & $(4.09)$ & $(0.00)$ & $(2.85)$ & $(1.04)$ & $(1.02)$ \\
\hline
\end{tabular}




\section{Conclusions}

In this paper, we have proposed a novel method to avoid local optima in a clustering process. The essential concept is to constrain the descent direction for an objective function by the calculus of variations. We apply this concept to a robust $l_{2 \mathrm{~m}}-\mathrm{FCM}$ algorithm. The idea of the $l_{2 \mathrm{~m}^{-}}$ FCM algorithm is to drive the cluster centers to the medians of the data set. It is robust to noise and outperforms many existing methods [18]. By imposing this constraint, the proposed method is able to avoid trapping to local optima and performs better than the $l_{2 \mathrm{~m}}-\mathrm{FCM}$ algorithm. Our experiments show that the proposed algorithm has a higher stability than the $l_{2 \mathrm{~m}}-\mathrm{FCM}$ algorithm. We have also compared the proposed method with seven other clustering methods using both synthetic and real world data sets. The results show that the proposed method outperforms these algorithms and is able to yield good results. In the future, we will apply the technique based the calculus of variations to other clustering algorithms to avoid local optimal solutions.

Acknowledgement: This work is supported by two grants from the Hong Kong Research Grant Council (Projects CityU1035/02E and CityU122005).

\section{Appendix A}

Theorem 1:

$$
\text { Let } \mathrm{E}_{\varepsilon}(\mathbf{v})=\sum_{i=1}^{n} \sqrt{\varepsilon^{2}+\left\|\mathbf{x}_{i}-\mathbf{v}\right\|_{2}^{2}},
$$

which is the objective function $\mathrm{J}(\mathrm{U}, \mathrm{V} ; \mathbf{X})$ for $c=1$. Assume that $\mathrm{E}_{\varepsilon}(\mathbf{v})$ is a $\mathrm{C}^{1}$ function, which is a continuous differentiable function. If $\mathbf{u}$ and $\mathbf{v}$ are the minimum of $\mathrm{E}_{0}(\mathbf{u})$ and $\mathrm{E}_{\varepsilon}(\mathbf{v})$ respectively, then $\|\mathbf{u}-\mathbf{v}\|_{2} \leq 2|\varepsilon|$.

Proof: Without loss of generality, we denote $\mathbf{u}=\gamma \varphi$ and $\mathbf{v}=\beta \varphi$ as the minimums of $\mathrm{E}_{0}(\mathbf{u})$ and $\mathrm{E}_{\varepsilon}(\mathbf{v})$ respectively, where $\gamma=0, \beta>0$ are given scalar values and $\boldsymbol{\varphi \neq 0}$ is a given unit vector. Our goal is to prove that the upper bound of $\|\mathbf{u}-\mathbf{v}\|_{2}=|\beta-\gamma|=|\beta|$ is $2|\varepsilon|$. Putting $\mathbf{v}=\alpha \varphi$, we have

$$
\mathrm{E}_{\varepsilon}(\alpha)=\sum_{i=1}^{n} \sqrt{\varepsilon^{2}+\left\|\mathbf{x}_{i}-\alpha \varphi\right\|_{2}^{2}} .
$$

As $\gamma$ is the minimum, it satisfies the following equation.

$$
\frac{\mathrm{dE}_{\varepsilon}(\alpha)}{\mathrm{d} \alpha}=\sum_{i=1}^{n} \frac{\alpha-\mathbf{x}_{\mathrm{i}}^{\mathrm{T}} \varphi}{\sqrt{\varepsilon^{2}+\left\|\mathbf{x}_{i}-\alpha \varphi\right\|_{2}^{2}}}=0 \quad \text { for } \quad \alpha=\gamma
$$

We assume the following inequality holds.

$$
\left.\frac{\mathrm{dE}_{\varepsilon}(\alpha)}{\mathrm{d} \alpha}\right|_{\alpha=0}=\sum_{i=1}^{n} \frac{-\mathbf{x}_{\mathrm{i}}^{\mathrm{T}} \varphi}{\sqrt{\varepsilon^{2}+\left\|\mathbf{x}_{i}\right\|_{2}^{2}}} \geq 0 .
$$

If this inequality does not hold, we take $\mathbf{y}_{\mathrm{i}}=-\mathbf{x}_{\mathrm{i}}$ and $\eta=-\gamma$. Then, $\eta \varphi$ is the minimum of $\left\{\mathbf{y}_{\mathrm{i}}\right\}$. This is shown below.

$$
0=\sum_{i=1}^{n} \frac{\gamma-\mathbf{x}_{\mathrm{i}}^{\mathrm{T}} \varphi}{\sqrt{\varepsilon^{2}+\left\|\mathbf{x}_{i}-\gamma \varphi\right\|_{2}^{2}}}=\sum_{i=1}^{n} \frac{-\eta+\mathbf{y}_{\mathrm{i}}^{\mathrm{T}} \varphi}{\sqrt{\varepsilon^{2}+\left\|\mathbf{y}_{i}-\eta \varphi\right\|_{2}^{2}}}=\sum_{i=1}^{n} \frac{\eta-\mathbf{y}_{\mathrm{i}}^{\mathrm{T}} \varphi}{\sqrt{\varepsilon^{2}+\left\|\mathbf{y}_{i}-\eta \varphi\right\|_{2}^{2}}} .
$$

By substituting $\mathbf{y}_{\mathrm{i}}=-\mathbf{x}_{\mathrm{i}}$ and $\eta=-\gamma$ into Equation (A4), the quantity will be positive again. 


$$
\sum_{i=1}^{n} \frac{-\mathbf{y}_{i}^{\mathrm{T}} \varphi}{\sqrt{\varepsilon^{2}+\left\|\mathbf{y}_{i}\right\|_{2}^{2}}} \geq 0 .
$$

Thus, we assume that Equation (A4) holds.

Next, we prove that the following quantity is positive.

$$
\left.\frac{\mathrm{dE}_{\varepsilon}(\alpha)}{\mathrm{d} \alpha}\right|_{\alpha=2 \varepsilon}=\sum_{i=1}^{n} \frac{2 \varepsilon+\mathbf{x}_{\mathrm{i}}^{\mathrm{T}} \varphi}{\sqrt{\varepsilon^{2}+\left\|\mathbf{x}_{i}+2 \varepsilon \varphi\right\|_{2}^{2}}} .
$$

We differentiate Equation (A7) with respect to $\varepsilon$.

$$
\begin{aligned}
& \frac{\mathrm{d}}{\mathrm{d} \varepsilon}\left(\left.\frac{\mathrm{dE}_{\varepsilon}(\alpha)}{\mathrm{d} \alpha}\right|_{\alpha=2 \varepsilon}\right) \\
= & \frac{\mathrm{d}}{\mathrm{d} \varepsilon} \sum_{i=1}^{n} \frac{2 \varepsilon+\mathbf{x}_{\mathrm{i}}^{\mathrm{T}} \varphi}{\sqrt{\varepsilon^{2}+\left\|\mathbf{x}_{i}+2 \varepsilon \varphi\right\|_{2}^{2}}} \\
= & \sum_{i=1}^{n} \frac{4 \varepsilon^{2}+3 \varepsilon \mathbf{x}_{\mathrm{i}}^{\mathrm{T}} \varphi+\left\|\mathbf{x}_{i}\right\|_{2}^{2}+\left\|\mathbf{x}_{i}\right\|_{2}^{2}-\left(\mathbf{x}_{\mathrm{i}}^{\mathrm{T}} \varphi\right)^{2}}{\left(\sqrt{\varepsilon^{2}+\left\|\mathbf{x}_{i}+2 \varepsilon \varphi\right\|_{2}^{2}}\right)^{3}} \\
= & \sum_{i=1}^{n} \frac{\left\|\mathbf{x}_{i}+(3 / 2) \varepsilon \varphi\right\|_{2}^{2}+(4-9 / 4) \varepsilon^{2}+\left\|\mathbf{x}_{i}\right\|_{2}^{2}-\left(\mathbf{x}_{\mathrm{i}}^{\mathrm{T}} \varphi\right)^{2}}{\left(\sqrt{\varepsilon^{2}+\left\|\mathbf{x}_{i}+2 \varepsilon \varphi\right\|_{2}^{2}}\right)^{3}} \\
> & 0
\end{aligned}
$$

As the derivative of Equation (A7) is positive, the function is monotonic increasing.

$$
\sum_{i=1}^{n} \frac{2 \varepsilon+\mathbf{x}_{\mathrm{i}}^{\mathrm{T}} \varphi}{\sqrt{\varepsilon^{2}+\left\|\mathbf{x}_{i}+2 \varepsilon \varphi\right\|_{2}^{2}}} \geq \sum_{i=1}^{n} \frac{\mathbf{x}_{\mathrm{i}}^{\mathrm{T}} \varphi}{\sqrt{\left\|\mathbf{x}_{i}\right\|_{2}^{2}}}=0
$$

as $\mathbf{u}=0$ is the minimum of $\mathrm{E}_{0}(\mathbf{u})$. By combining the Equation (A4), we have the following conclusion.

$$
\sum_{i=1}^{n} \frac{-2 \varepsilon-\mathbf{x}_{\mathrm{i}}^{\mathrm{T}} \varphi}{\sqrt{\varepsilon^{2}+\left\|\mathbf{x}_{i}-(-2 \varepsilon) \varphi\right\|_{2}^{2}}} \leq 0 \leq \sum_{i=1}^{n} \frac{-\mathbf{x}_{\mathrm{i}}^{\mathrm{T}} \varphi}{\sqrt{\varepsilon^{2}+\left\|\mathbf{x}_{i}\right\|_{2}^{2}}} .
$$

As $E_{\varepsilon}(\mathbf{v})$ is a $C^{1}$ function and by intermediate value theorem, we can always find a quantity $|\beta|<2|\varepsilon|$ such that the following equation holds.

$$
\sum_{i=1}^{n} \frac{\beta-\mathbf{x}_{\mathrm{i}}^{\mathrm{T}} \varphi}{\sqrt{\varepsilon^{2}+\left\|\mathbf{x}_{i}-(\beta) \varphi\right\|_{2}^{2}}}=0 .
$$

That is, the solution $\mathrm{E}_{\varepsilon}(\mathbf{v})$ is smaller than $2|\varepsilon| \cdot \square$

\section{Appendix B}

The goal of calculus of variations is to find an equation so that the optimal solution of the objective function is satisfied. The calculus of variations is formulated as follows. Given the optimal solution $\mathbf{v}^{*}(\mathrm{~s})$ of the objective function $\mathrm{J}(\mathbf{v}(\mathrm{s}))$, the function value at perturbed $\mathbf{v}^{*}(\mathrm{~s})$ is 
larger than the value at $\mathbf{v}^{*}(\mathrm{~s})$. That is, $\mathrm{J}\left(\mathbf{v}^{*}(\mathrm{~s})\right) \leq \mathrm{J}\left(\mathbf{v}^{*}(\mathrm{~s})+\gamma \phi(\mathrm{s})\right)$, where $\gamma$ is a scalar and $\phi(\mathrm{s})$ is an arbitrary function. This implies that the function $\mathrm{J}\left(\mathbf{v}^{*}(\mathrm{~s})+\gamma \phi(\mathrm{s})\right)$ reaches minimum at $\gamma=0$, and thus

$$
\left.\frac{\mathrm{d}}{\mathrm{d} \gamma}\right|_{\gamma=0} \mathrm{~J}(\mathrm{v} *(\mathrm{~s})+\gamma \phi(\mathrm{s}))=0
$$

We illustrate the application of calculus of variations to the FCM algorithm. The FCM algorithm can be formulated as follows.

$$
J(\mathbf{v}(s))=\sum_{i=1}^{n} \int_{\Omega} \mu_{i}^{m}(s)\left\|\mathbf{x}_{i}-\mathbf{v}(s)\right\|_{\varepsilon} H(s) d s
$$

where $\Omega=[0,1]$ and $H(s)$ is a delta function. $H(s) \rightarrow \infty$ if $s \in\left\{s_{\mathrm{k}}: 0 \leq \mathrm{s}_{\mathrm{k}} \leq 1, k=1,2, \ldots c\right\}$, otherwise, $\mathrm{H}(\mathrm{s})=0$. We apply the calculus of variations to this objective function with an arbitrary function $\phi(\mathrm{s})$. Taking the derivative with respect to $\gamma$ at $\gamma=0$, we have

$$
\begin{aligned}
0 & =\left.\frac{d}{d \gamma}\right|_{\gamma=0} J(\mathbf{v}(s)+\gamma \phi(s)) \\
& =\left.\frac{d}{d \gamma}\right|_{\gamma=0} \sum_{i=1}^{n} \int_{\Omega} \mu_{i}^{m}(s)\left\|\mathbf{x}_{i}-\mathbf{v}(s)-\gamma \phi(s)\right\|_{\varepsilon} H(s) d s \\
& =\sum_{i=1}^{n} \int_{\Omega} \mu_{i}^{m}(s) \frac{\mathbf{v}(s)-\mathbf{x}_{i}}{\left\|\mathbf{v}(s)-\mathbf{x}_{i}\right\|_{\varepsilon}} \phi(s) H(s) d s
\end{aligned}
$$

As $\phi(s)$ is an arbitrary function, and $\mathrm{H}(\mathrm{s})$ is a delta function, which is not equal to zero only at a finite number of points. We have

$$
\sum_{i=1}^{n} \mu_{i k}^{m} \frac{\mathbf{v}_{k}-\mathbf{x}_{i}}{\left\|\mathbf{v}_{k}-\mathbf{x}_{i}\right\|_{\varepsilon}}=0
$$

which is the updating equation for the $l_{2 \mathrm{~m}}$-FCM algorithm.

\section{References}

[1] S. Chu, J. DeRisi, M. Eisen, J. Mulholland, D. Botstein, P. Brown, I. Herskowitz, "The Transcriptional Program of Sporulation in Budding Yeast," Science, Vol. 282, pp. 699-705, 1998.

[2] R. Cho, M. Campbell, E. Winzeler, L. Steinmetz, A. Conway, L. Wodicka, T. Wolfsberg, A. Gabrielian, D. Landsman, D. Lockhart and R. Davis, "Genome-wide transcriptional analysis of the mitotic cell cycle," Molecular Cell, Vol. 2, pp. 65-78, 1998.

[3] H. Agrawal, "Extreme Self-Organization in Networks Constructed from Gene Expression Data," Physical Review Letters, Vol. 89, pp. 268702 [4 pages], 2002.

[4] J. C. Bezdek, Pattern Recognition With Fuzzy Objective Function Algorithms, Plenum, New York, 1981.

[5] R. A. Johnson, D. W. Wichern, Applied Multivariate Statistical Analysis, 5th ed, Upper Saddle River, N.J.: Prentice Hall, 2002.

[6] S. C. Chu, J. F. Roddick, T. Y. Chen and J. S. Pan, "Efficient search approaches for K-medoids-based algorithms," Proceedings. 2002 IEEE Region 10 Conference on Computers, Communications, Control and Power Engineering, Vol. 1, pp. $712-715,2002$.

[7] E. Gokcay and J. C. Principe, "Information theoretic clustering," IEEE Transactions on Pattern Analysis and Machine Intelligence, Vol. 24, no. 2, pp. 158 -171, 2002.

[8] L. Kaufman and P. J. Rousseeuw, Finding Groups In Data: An Introduction To Cluster analysis, New York: Wiley, 1990.

[9] A. K. Jain, M. N. Murty and P. J. Flynn, "Data clustering: a review," ACM Computing Surveys, Vol. 31, no. 3 pp. 264-323, 1999. 
[10] T. Kohonen, Self-organization and associative memory, 2nd ed., Berlin: Springer-Verlag, 1988.

[11] F. Hoppner, F. Klawonm, R. Kruse and T. Runkler, Fuzzy cluster analysis: methods for classification, data analysis, and image recognition, Chichester: John Wiley, 1999.

[12] V. Barnett and T. Lewis, Outliers in Statistical data, 3rd edition, John Wiley, 1994.

[13] S.Z. Selim and K. Alsultan, "A simulated annealing algorithm for the clustering problem," Pattern recognition, Vol. 24, pp. 1003-1008, 1991

[14] X. Y. Wang and J. M. Garibaldi, "Simulated annealing fuzzy clustering in cancer diagnosis," Informatica, Vol. 29, pp. 61-70, 2005.

[15] K. Krishna, M. M. Narasimha Murty, "Genetic K-means algorithm," IEEE Transactions on Systems, Man and Cybernetics, Part B, Vol. 29, Issue 3, pp. 433-439, 1999.

[16] L. C. Evans, Partial Differential Equations, Providence, R.I.: American Mathematical Society, 1998.

[17] L. L. Richard, Introductory quantum mechanics, 3rd edition, Reading, Mass.: Addison-Wesley Pub., 1998

[18] B. S. Y. Lam and H. Yan, "Robust Clustering Algorithm for Suppression of Outliers," Int'l Symp. Intel. Multimedia, Video \& Speech Processing, pp 691-694, 2004.

[19] B. Lam and H. Yan, "Improved Clustering Algorithm Based on Calculus of Variation," ICPR 2006, to appear, 2006.

[20] K. L. Wu and M. S. Yang, "Alternative c-means clustering algorithms," Pattern recognition, Vol.35, pp.22672278, 2002.

[21] J. D. Banfield and A. E. Raftery, "Model-based Gaussian and non-Gaussian clustering," Biometrics, Vol. 49, pp. 803-821, 1993.

[22] M. Barni, V. Cappellini and A. Mecocci, "Comments on a possibilistic approach to clustering, " IEEE Transactions on Fuzzy Systems, Vol. 4, no. 3, pp. 393-396, 1996.

[23] R. Krishnapuram and J. M. Keller, "A possibilistic approach to clustering," IEEE Transactions on Fuzzy Systems, Vol. 1, no. 2, pp. 98-110, 1993.

[24] http://www.ics.uci.edu/ mlearn/MLSummary.html

[25] http://cellcycle-www.stanford.edu/

[26] http://cmgm.stanford.edu/pbrown/sporulation

[27] S. Datta and S. Datta, "Comparisons and validation of statistical clustering techniques for microarray gene expression data," Bioinformatics, Vol. 19, no. 4, pp. 459-466, 2003. 